\title{
Original
}

\section{El peritaje forense en los casos de violación en Honduras previo a la independencia}

\section{Forensic expertise in rape cases in Honduras before the independence \\ José Manuel Cardona Amaya ${ }^{1 *}$ (D) https://orcid.org/0000-0003-4870-5619}

${ }^{1}$ Departamento de Historia, Universidad Nacional Autónoma de Honduras

*Autor de correspondencia: jmcardona@unah.edu.hn

\section{Palabras clave}

Violación, Partería, Himen, Honduras.

\section{Keywords}

Rape, Midwifery, Hymen Honduras.

\section{Historia del artículo}

Recepción: 02 marzo 2021

Aprobación: 30 abril 2021

DOI: https://doi.org/10.5377/ rcfh.v7i1.11584

Citar como: Amaya JMC. El peritaje forense en los casos de violación en la Honduras previo a la Independencia. Rev. cienc. forenses Honduras. 2021; 7(1): 214. DOI: https://doi.org/10.5377/ rcfh.v7i1.11584

Declaración de relaciones y actividades financieras $y$ conflictos de interés: ninguna.

\section{RESUMEN}

Justificación: la bibliografía sobre delitos sexuales en el periodo de dominación hispánica en América (1492-1821) es escasa, existiendo importantes vacíos historiográficos que dificultan la reconstrucción de un panorama general del proceso penal conducido en esa época, por lo que, investigar el peritaje conducido en los casos de violación es un primer paso para rastrear la evolución del tratamiento de esos casos en las instituciones judiciales. Objetivo: describir cómo se ejecutaba el peritaje forense en los casos de violación sucedidos en la Honduras previo a su independencia política de España. Metodología: se adoptó un enfoque cualitativo con un diseño exploratorio, utilizándose fuentes primarias que fueron seleccionadas mediante muestreo no probabilístico. Resultados: se logró identificar dos casos en los cuales se hizo recurso de peritos para confirmar una violación. En ambos las expertas tenían el oficio de partera, brindando declaraciones que reflejan una falta de preparación profesional y un conocimiento basado en la experiencia. Las declaraciones fueron transcritas conservándose la ortografía de la época y comentadas para ubicarlas en su contexto. Conclusión: en la Honduras de los años previos a la independencia se realizaba un peritaje forense en casos de violación basado en la experiencia laboral de los peritos y no en información científica o preparación académica, esto condujo a que los juicios de los expertos se vieran afectados por sus sesgos culturales. 


\section{ABSTRACT}

Justification: the bibliography on sexual crimes in the period of Hispanic domination in America (1492-1821) is scare, there are important historiographic gaps that make it difficult to reconstruct a general panorama of the criminal process conducted at that time, therefore, investigating the expert forensic opinion conducted in rape cases is a first step in tracking the evolution of the treatment of these cases in judicial institutions. Objective: to describe how forensic expertise was carried out in rape cases that occurred in Honduras before its political independence from Spain. Methodology: a qualitative approach was adopted with an exploratory design, using primary sources that were selected by non-probabilistic sampling. Results: it was possible to identify two cases in which an appeal was made by experts to confirm a rape. In both, the experts had the profession of midwife, providing statements that reflect a lack of professional preparation and knowledge based on experience. The statements were transcribed, keeping the spelling of the time and commented on, to place them in context. Conclusion: in Honduras before the independence, a forensic expert opinion was carried out in rape cases based on the work experience of the experts and not on scientific information or academic preparation, this led to the experts' judgments being affected by their cultural biases.

\section{INTRODUCCIÓN}

En Honduras, la historia de los delitos sexuales es un campo todavía sin explotar, existiendo apenas un artículo científico sobre la materia ${ }^{1}$. En el resto de Latinoamérica, la bibliografía es también reducida, habiendo selectos estudios para México ${ }^{2,3,4,5}$, Chile $^{6}$, Costa Rica $^{7,8}$, Colombia $^{9}$ y Venezuela ${ }^{10}$. En cuanto al peritaje forense emprendido para verificar el hecho, solamente tres artículos han abordado la temática en el periodo histórico del dominio del Imperio Español en América ${ }^{11,12,13}$. Este vacío historiográfico previene la discusión holística de la historia de los delitos sexuales, careciendo los investigadores de suficiente bibliografía para reconstruir las causas de estos crímenes y exponer el procedimiento judicial emprendido en la época. Por lo tanto, la presente investigación se propone describir cómo se conducía el peritaje forense en los casos de violación en la Honduras colonial, específicamente en los años previos a la independencia política de la provincia, con el propósito de contribuir a la historiografía sobre la materia.

Tratándose de una investigación histórica, se utilizó un enfoque cualitativo con un diseño exploratorio, por tratarse de un tema inédito en la historiografía nacional. Las fuentes de 
información fueron expedientes penales del periodo colonial de Honduras (1524-1821), obtenidas en el Archivo Nacional de Honduras. La selección de los casos se hizo mediante el muestreo por conveniencia, una técnica no probabilística que ha cedido un total de dos expedientes con los criterios necesarios para su análisis. En específico, se usaron aquellos procesos penales por violación en los cuales el juez había solicitado realizar el peritaje forense en la víctima.

De especial atención resultaron los testimonios de las personas convocadas para ejecutar el peritaje, los cuales han sido transcritos fielmente, conservándose la ortografía de la época. Estas declaraciones proveyeron una ventana a las técnicas utilizadas hace dos siglos en Honduras para detectar una violación, además de ofrecer valiosa información sobre la mentalidad del periodo. Estos datos, si bien corresponden a un tiempo pasado de la historia de Honduras, invitan a reflexionar sobre qué tanto se ha avanzado en materia de atención a las víctimas en casos de abuso sexual y cuánto falta aún por resolver.

\section{METODOLOGÍA}

En esta investigación se adoptó un enfoque cualitativo, definiéndose este como aquel que utiliza datos no cuantitativos para brindar una interpretación basada en el contexto social, económico, político e histórico del objeto estudiado ${ }^{14}$. El diseño de la investigación fue de corte exploratorio, que se comprende como aquel que realiza un primer acercamiento a un tema para abrir el debate científico sobre este y promover su discusión ${ }^{15}$.

Para obtener los datos se utilizaron fuentes primarias que son aquellas contemporáneas a la delimitación temporal y espacial de determinado objeto de estudio ${ }^{16}$. En específico, se tomaron expedientes del Archivo Nacional de Honduras, entidad que resguarda documentación de los siglos XVII al XX. La selección de los expedientes se hizo mediante el muestreo por conveniencia, siendo este una técnica no probabilística en la cual se escogen los casos que presenten las características de inclusión deseables para el estudio $^{17}$. A partir de esto, se hizo lectura del índice del periodo colonial del Archivo Nacional de Honduras y se seleccionaron los casos de violación, de lo cual resultaron siete expedientes. Posteriormente, se leyó el contenido de los casos, descartándose todos aquellos en los cuales no se practicó un peritaje forense sobre las víctimas de violación, desechándose así cinco expedientes y restando dos, con los cuales se realizó la descripción. Ya que ambos documentos resultantes de la aplicación de los criterios de selección poseen una fecha cercana a la independencia política de Honduras con España 
en 1821, este artículo refleja solamente las prácticas vigentes a inicios del siglo XIX en Honduras y no la totalidad del periodo de dominio hispánico en el territorio.

Debido a que la información expuesta en esta investigación antecede por siglos a su realización, se ha empleado el método históricológico para brindar inferencias de la cultura y la sociedad de la época, enriqueciendo así la exposición de los hallazgos. Este método se refiere a la formulación de inferencias lógicas del objeto estudiado, utilizando el conocimiento previo sobre la sociedad y la cultura en que este se desarrolló ${ }^{18}$. Esto hizo posible comprender los motivos porqué se practicaban los peritajes forenses en los casos de violación de ciertas maneras y no de otras.

\section{RESULTADOS}

Habiendo revisado siete expedientes de procesos de violación del Archivo Nacional de Honduras, fue posible identificar dos casos en los cuales los jueces solicitaron la realización de un peritaje forense. Que solamente en una cantidad mínima de los procesos se haya solicitado dicho peritaje, podría explicarse por la falta de profesionales capacitados para el análisis. En el caso específico de los casos de estupro o violación, la selección de los peritos y la validez de su testimonio presentaba problemas, como aclara Ortiz: "La selección de las mujeres aptas para realizar un peritaje debía ser sumamente cuidadosa, pero a pesar de toda precaución, las dudas acerca de la autenticidad y honestidad de sus testimonios se pusieron en entredicho con bastante frecuencia, en parte porque los cirujanos contribuían a extender el descrédito"19. También debe tomarse en cuenta que los procesos penales indianos dependían de la prueba testimonial, por lo que, en la mayoría de casos bastaba con pasar a declarar a las partes. Esta última explicación se refuerza con las particularidades de los casos identificados: en el primero, la víctima tenía nueve años, mientras que, en el segundo, la agredida contaba con ocho años.

Ambos casos identificados se dieron en la segunda década del siglo XIX, pocos años antes de la Independencia de Honduras con respecto a España. El primer caso se suscitó en 1816, cuando S. Pineda, una niña de nueve años fue violada por M. Jiménez de 30 años en Goascorán. El segundo caso en el cual se practicó un examen a la víctima se da en 1819 en Tegucigalpa, llamándose la agredida I. Romero, de ocho años y el agresor R. Flores de 25 años. En ninguno de los expedientes se consignó la etnicidad de las víctimas o los agresores.

El delito de estupro se definía como la 
penetración forzosa de la víctima, siempre y cuando esta fuera doncella o poseyera "honor"7. Es por esto que las causas judiciales estaban encausadas no a comprobar la acción criminal sobre la mujer, si no a verificar que esta estuviera virgen. Según Bazán ${ }^{20}$, una mujer que había perdido su "honor" se arriesgaba a perder la salvación de su alma, su posición social, su herencia y la oportunidad a un matrimonio ventajoso. Por lo que los jueces debían de asegurarse con personas entendidas en la anatomía genital de las mujeres para ejecutar estos peritajes. Esto también quiere decir que un cirujano o barbero empírico no era el adecuado en estos casos, por carecer de larga trayectoria que una partera tenía con su campo de especialidad.

En ambos casos, el juez convocó a dos mujeres por separado para realizar el peritaje, poseyendo ambas el oficio de "parteras". Las mujeres que atendieron el primer caso fueron S. Hernández y J. Castro, dos señoras de la misma localidad de la víctima. El segundo caso fue abordado por $\mathrm{M}$. Núñez de 60 años y por JM Núñez de 55 años, habitando ambas en la Villa de Tegucigalpa. Los expedientes no aportaron más datos sobre estas mujeres, ni de su casta, ni de sus años de experiencia.

En el periodo estudiado, la convocatoria a los peritajes se hacía con base en dos legislaciones: Las Partidas del Rey Alfonso, "El Sabio" y en la Recopilación de las leyes de Castilla ${ }^{21}$. En el primero, se establecía que los jueces podían convocar "consejeros" para que diesen su opinión sobre asuntos en los que el togado desconociere. En el segundo, se limitaba al nombramiento de estos peritos "para en cosa que consiste en cuenta o tasación, o pericia de persona o arte" 21 .

En los documentos de los casos se consignó la declaración que estas mujeres dieron sobre el peritaje, representando estas una importante ventana al proceso probatorio de la justicia pre independentista hondureño. En el caso de S. Pineda, de 1816, el testimonio de ambas expertas se registró en un mismo pasaje, transcribiéndose a continuación, respetándose la ortografía de la época:

"Recivi [sic] juramento en toda forma a $S$ Hernanz [sic] y a J Castro baxo [sic] cuya gravedad haviendo [sic] verificado el reconosimto [sic] an [sic] dado en el auto anterior expucieron [sic]: que haviendolo [sic] practicado con el mayor escrúpulo hallaron qe [sic] S [sic] no está violada pero se conose [sic] que se intentó el extrupo [sic] de cuyas resultas tiene en el exterior de las partes genitales una lastimadura que se conose [sic] fue hecha en el acto esto dixeron [sic] y firmó la que supo conmigo". ${ }^{22}$.
Rev. cienc. forenses Honduras, Volumen 7, N 1, Año 2021

Open Access

(C)Autor(es)
URL: http://www.bvs.hn/RCFH/html5/

CC-BY-NC 4.0 internacional 
En el pasaje anterior se aprecia como ambas mujeres llamadas a conducir el peritaje llegaron a la conclusión de que la niña no había sido violada. Habiendo examinado las partes íntimas de la víctima, las expertas declararon que no hubo penetración, pero que en las partes exteriores del área vaginal habían detectado una herida, comprobándose así el intento criminal.

En el caso de I. Romero, sucedido en 1819, las parteras dieron declaraciones individuales. A continuación, se transcribe lo expuesto por $M$. Núñez:

"Haviendo [sic] comparecido M. Núñez, soltera q.e [sic] doy fe conosco [sic], de edad de secenta [sic] años, y recibido juramto [sic] que hizo por Dios ntro [sic]. Señor y una señal de la cruz, por el que ofreció decir verdad en lo que supiere, y se le fuese preguntado y siendolo [sic] por la integridad de $Y$. [sic] J. Romero en la parte de la generación respondió que esta tan buena como lo estaría antes que como hace tiempo que la que declara hoyo [sic] decir havia [sic] cometido el extrupo [sic] ha soldado, y a ella le consta por haver [sic] curado a una criatura que habiéndose caído de un palo y rompidose [sic] la parte de la criacion [sic], la curo y quedo tan buena como pudo haver [sic] estado antes" 23.

Como se aprecia, la partera aseguró que, aunque la víctima sí había sido penetrada, esta había "soldado", es decir, que la ruptura del himen se había reparado por sí misma. Para dar más peso a su declaración, M. Núñez hizo recurso a su experiencia previa, afirmando que en una ocasión anterior ella había atendido un caso similar. La otra experta declaró similarmente, como se transcribe a continuación:

"Ofreció decir verdad en lo que supiese y fuese preguntado, y siéndolo pr. [sic] la integridad de $Y$ [sic] J. Romero en la parte de la generación, dijo: que haviendola [sic] registrado la encontró buena, que no hay duda habría soldado. Preguntándole que si llegavan [sic] ha [sic] soldar siempre que se dañaban la parte, dijo que no sabe; pero que Y. [sic] estaba tan buena como lo estaría antes". ${ }^{23}$

Si bien estas declaraciones no evidencian que las parteras hayan poseído conocimientos técnicos de anatomía humana, sí demuestran que estas habían adquirido métodos de identificar irregularidades a lo largo de su labor profesional. A pesar de no ser realizado por personas con preparación profesional, que este tipo de peritaje haya sido solicitado por un juez, demuestra su importancia en el proceso, sobre todo, considerándose que las víctimas en ambos casos eran menores de edad.

En cuanto a si los peritajes influyeron en el resultado de los casos, no se encontró suficiente información para poder hacer esta afirmación. 
En el caso de S. Pineda, el acusado logró que el tío de la víctima le otorgara un perdón antes de dictada la sentencia, por lo que, siguiendo el procedimiento de la época, el juez liberó al agresor sin culminar el proceso. En el segundo caso, mientras el acusado esperaba sentencia en la cárcel de Tegucigalpa, se le concedió la gracia de indulto mediante recurso interpuesto en la Real Audiencia de Guatemala, quedando así librado.

\section{DISCUSIÓN}

Los datos encontrados evidencian una influencia de las creencias culturales en el proceso penal por violación. En primera instancia, que las personas llamadas a practicar el peritaje de las partes íntimas de las víctimas hayan sido mujeres, con el oficio de partera, se explica porque las mismas víctimas eran mujeres. Sidy, aclara sobre este punto que "les correspondía a las parteras hacerse cargo de la observación exhaustiva de los cuerpos femeninos." ${ }^{12}$. Entendiéndose por esto una delimitación entre quién debía de observar qué tipo de cuerpos. Según Tortorici ${ }^{13}$, el conocimiento que las parteras tenían del cuerpo de la mujer era lo que las hacía los peritos preferenciales en estos casos, como se indica en el siguiente pasaje: "it was this intimate experiential knowledge of the female body, obstetrics, and child birth that enabled midwives - usually older women who were often widowed-to participate as medical examiners"13. Es por esta categoría de género que se prefería a las parteras por sobre los cirujanos o médicos empíricos.

En cuanto a los hospitales y su personal, estos no jugaban ningún rol en el peritaje forense en este periodo. Los hospitales de Honduras habían sido construidos y mantenidos por las Órdenes religiosas y como tal respondían a la concepción de "hospital" que tenía la Iglesia Católica desde la Edad Media. Sobre este punto, Muriel aclara que "el concepto moderno de lo que es un hospital es tan diferente al de aquellos tiempos, que se impone una reflexión. Muchas de las obras, en bien de los menesterosos, se realizaban entonces, tenían la denominación común de "hospitales", pues en ellos la palabra y la institución misma tenían una enorme amplitud"24. En la América hispana, los hospitales fueron fundados bajo esta misma idea de la caridad, como un lugar para resguardar a los enfermos y propiciarles los cuidados respectivos y no, como un centro profesional en el cual los expertos de la medicina ejercerían su profesión ${ }^{24}$. Un ejemplo claro de esto es el hospital San Juan de Dios de Comayagua, que para el año 1783 contaba solamente con un pequeño cuarto en el cual dormían los enfermos, una capilla, una sacristía y un camposanto. Es por esta razón que la existencia o inexistencia de los 
hospitales en Honduras no se relaciona con la disponibilidad de peritos en los casos judiciales ${ }^{25}$. También se debe observar que estos servicios se encontraban centralizados en los principales centros urbanos ${ }^{26}$, limitando el acceso que las personas tenían a estos.

Debido a que en este periodo la violación y el estupro se tipificaban dentro los llamados "delitos contra el honor"27, en algunos casos no se pasaba a la verificación del crimen por llegarse a un acuerdo monetario entre las partes; los "delitos contra el honor" no eran considerados de gravedad, en algunos casos eran resueltos in situ por los tenientes de partido, sin remitir a las partes a los centros urbanos en donde sí se podía solicitar realizar un peritaje. Esto puede explicar la ausencia de profesionales de la medicina en algunos procesos judiciales de la época.

Ya que las mujeres que ejecutaban el peritaje no contaban con conocimientos profesionales de medicina, su opinión se basaba en la experiencia $^{28}$ Al basarse en observación pragmática, estas parteras podían cometer errores de asociación gravísimos, que comprometiesen sus declaraciones. Un ejemplo de esto fue la declaración de Marta Núñez en el caso de I. Romero, de 1819. En esa ocasión, la declarante afirmó que el himen de la niña violada se había reparado solo, basándose en que anteriormente, ella había atendido un caso de otra fémina que, habiendo caído de un árbol, se había roto sus partes íntimas y recuperado de este daño con el tiempo. Aunque pueda parecer inverosímil relacionar una violación sexual con la caída de un árbol, para estas parteras que nunca habían estudiado medicina, era una analogía perfectamente aceptable.

Los expedientes no aportaron datos para hacer observaciones sobre las diferencias en la aplicación de la justicia entre castas raciales ni grupos económicos. Esto se debió a dos factores: la escasa documentación de la época y la poca importancia de estas variables para los jueces. No embargante, se espera que esta primera aproximación a la temática dé paso a nuevas investigaciones que puedan resolver esta interrogante.

Un aspecto importante que revelan los casos es la valoración de la prueba. El proceso penal indiano era eminentemente testimonial ${ }^{29}$, debiendo el juez pasar a ambas partes a declarar. Sin embargo, en los dos casos estudiados en esta investigación, la edad de las víctimas hacía que las declaraciones pasasen a segundo plano y se implementaba el peritaje forense para establecer los hechos. Esto no quiere decir que ambas niñas violadas no hayan pasado a dar su testimonio, sí lo hicieron, sino que, el juez, 
comprendiendo que por su edad no podrían explicar precisamente lo que les había sucedido, prefirió utilizar la opinión de los expertos para despejar sus dudas.

Los casos estudiados no brindan suficiente información para establecer la influencia que el peritaje forense en víctimas de violación tenía sobre la sentencia, aunque, sí es posible afirmar que las expertas declaraban siendo conscientes que su opinión afectaría el fallo del juez. Lo anterior se establece porque, en ambas ocasiones, las mujeres llamadas a realizar el peritaje se excedieron de sus propósitos iniciales. En vez de simplemente afirmar si había señal de una violación, estas mujeres se esforzaron por explicar al juez que las víctimas no habían sufrido daños irreparables o, que este ya había sanado. No se cuenta con la información para afirmar por qué las parteras hacían esto, pero se pueden inferir dos posibilidades: primero, que querían minimizar el daño psicológico en las agredidas, haciéndoles creer que la violación había sido algo pasajero que no dejaría secuelas físicas; segundo, que en la idiosincrasia de la época la violación no era un crimen de excesiva gravedad y que, por lo tanto, estas mujeres, minimizaban lo sucedido para evitar una condena al acusado.

\section{CONCLUSIONES}

Los expedientes de procesos penales por violación en la Honduras pre independentista hicieron posible describir el peritaje que se conducía para confirmar el hecho. Se encontró que las personas Ilamadas para hacer el examen eran mujeres con el oficio de partera, pertenecientes a la misma localidad de la víctima. Estas expertas no contaban con una educación profesional, sin embargo, su experiencia les permitía dar observaciones que eran tomadas en cuenta por el juez. No fue posible determinar el impacto positivo o negativo de la intervención de los peritos, por las circunstancias en que los casos estudiados se resolvieron, por lo que es necesario ubicar más fuentes en archivos extranjeros para poder aclarar este punto.

Aunque fue posible cumplir con el objetivo de la investigación, esta representa, solamente, un primer acercamiento al tema histórico del proceso penal y pericial por violación. Para poder contar con un panorama total de la evolución de los delitos sexuales en los juzgados hondureños, es necesario que los investigadores cubran todos los vacíos historiográficos, desde la llegada de los castellanos a América hasta el siglo XXI. Esto permitirá desentrañar raíces históricas que expliquen las causas de las debilidades de los sistemas actuales de este país y ofrecerá valiosos ejemplos para su mejora.

En la actualidad, la atención a víctimas de abuso
Rev. cienc. forenses Honduras, Volumen 7, N 1, Año 2021

Open Access $\quad$ CAutor(es).
URL: http://www.bvs.hn/RCFH/html5/

CC-BY-NC 4.0 internacional 
sexual ha evolucionado para incluir una serie de técnicas que permiten confirmar el hecho. Por ejemplo, en Honduras, se ha utilizado la cámara Gessell para obtener la información que permita emitir un dictamen forense sobre un caso $^{30}$. También los procedimientos han avanzado para evitar la confrontación de una víctima, evitando así la re-victimización que tanto daño hace la psique de la agredida ${ }^{31}$. A pesar de estos avances, la situación de la mujer entorno a un abuso sexual sigue siendo vulnerable. En el 2018, el Observatorio de Derechos Humanos reportó que el sistema de salud hondureño no cumplía con una atención integral a las víctimas, desde la emergencia médica al momento del hecho, hasta el tratamiento de las secuelas psicológicas después del mismo ${ }^{32}$. A lo anterior se suma una mora judicial en crecimiento, cuya lentitud atrapa a las mujeres en estos largos procesos, manteniendo viva la memoria de su sufrimiento ${ }^{32}$.

\section{REFERENCIAS BIBLIOGRÁFICAS}

1. Valladares OA. Sexo y represión en el período colonial en la Alcaldía Mayor de Tegucigalpa. Revista Estudios [Internet]. 2008 [citado 20 febrero 2021];(21): 33-40. Disponible en: https://revistas.ucr.ac.cr/index.php/estudios/ar ticle/view/23762/23929

2. Castañeda García C. Violación, estupro y sexualidad en la Nueva Galicia, 1790-1821.
México: Hexágono; 1989.

3. Baltazar Moscada D. Conflictos nupciales en los tribunales de justicia: estupro, adulterio y malos tratos. Estudios de caso en la ciudad de Santiago de Querétaro, 1779-1810. [Tesis de Maestría en Internet]. Querétaro: Universidad Autónoma de Querétaro;2018 [citado 13 abril 021]. Disponible en: http://ring.uaq.mx/handle/123456789/1037

4. Sánchez Pineda M. Como frágil hombre he pecado carnalmente. El delito de estupro forzoso en las ciudades de México y Valladolid, 17131810. [Tesis de Maestría en Internet]. Michoacán: Universidad Michoacana de San Nicolás de Hidalgo;2015 [citado 13 abril 2021]. Disponible en: http://bibliotecavirtual.dgb.umich.mx:8083/xml ui/handle/DGB UMICH/2439

5. Juárez Becerra I. Los mecanismos de género a través del acceso por fuerza en la Nueva Galicia del siglo XVIII. Vuelo Libre revista de Historia. [Internet]. 2016 [citado en 13 de abril de 2021];2(3):19-31. Disponible en: http://www.vuelolibre.revistadehistoria.cucsh.u dg.mx/sites/default/files/2 los mecanismos de genero a traves del acceso por fuerza 2a.p $\underline{\mathrm{df}}$

6. Valderrama NC. 'Ahora veremos lo que tiene esta niñita, El cuerpo como prueba de las violencias sexuales en el valle central de Chile, 1780-1830. Revista Historia y Justicia [Internet]. 
2018 [citado 20 febrero 2021];(11):1-32.

Disponible

en:

https://journals.openedition.org/rhi/4047

7. Rodríguez Sáenz E. Pecado, deshonor y crimen: el abuso sexual a las niñas: estupro, incesto y violación en Costa Rica (1800-1850, 1900-1950). Iberoamericana [internet]. 2002 [citado 20 febrero 2021];2(8):77-98. Disponible en:https://dialnet.unirioja.es/servlet/articulo?c odigo $=298963$

8. Rodríguez Sáenz E. Víctimas inocentes/amenazas corruptoras. Niñez “invención" del crimen juvenil y abuso sexual en Costa Rica (1800-1850 y 1900-1950). En: Sagot M, Díaz D, coordinadores. Antología del pensamiento crítico costarricense contemporáneo. Argentina: CLACSO; 2019. p. 299-332

9. Plata WE, Mendienta S. Delitos sexuales y contra la familia en el nororiente del Virreinato de la Nueva Granada, 1774-1810: de la norma a la aplicación. Historia y Espacio [Internet]. 2019 [citado 20 febrero 2021];15(52):109-136. Disponible

en:

\section{https://doi.org/10.25100/hye.v15i52.8206}

10. Manjarrés Ramos E. El dominio de las pulsiones del deseo: sexualidad e individuo en Venezuela del siglo XVI. Presente y Pasado [Internet]. 2017 [citado 20 febrero 2021];22(43):28-54. Disponible en: http://erevistas.saber.ula.ve/index.php/present eypasado/article/download/13910/2192192498 $\underline{9}$

11. Lorente Carpena A. Violación y transgresión: medicina forense y moral sexual en la España del siglo XIX. Asclepio [Internet]. 2010 [citado 20 febrero 2021];62(1): 177-208. Disponible en: http://asclepio.revistas.csic.es/index.php/asclep io/article/download/301/297/299

12. Sidy B. El cuerpo de Lorenza. Reflexiones en torno a un caso de estupro en el Río de la Plata (siglo xviii). Bol Antropol. [Internet]. 2021 [citado 13 abril 2021];36(61):58-77. Disponible en: https://revistas.udea.edu.co/index.php/boletin/ article/view/343380

13. Tortorici Z. Sexual violence, predatory masculinity and medical testimony in New Spain. Osiris [Internet]. 2015 [citado en 13 de abril de 2021];30(1):272-294. Disponible en: https://www.jstor.org/stable/10.1086/683023

14. Tracy S. Qualitative research methods: collecting evidence, crafting analysis communicating impact. 2a.ed. Estados Unidos de América: John Wiley-Blackwell; 2020.

15. Kale GV, Jayanth J. Introduction to research. En: Bairagi V, Munot MV, coordinadores. Research Methodology: a practical and scientific approach. Estados Unidos de América: Taylor and Francis; 2019.

16. Eiroa, M. El pasado en el presente: el conocimiento historiográfico en las fuentes digitales. Ayer [Internet]. 2018 [citado 20 febrero 
2021];110(2): 83-119. Disponible en: https://revistaayer.com/sites/default/files/artic $\underline{\text { ulos } / 110-3}$

ayer110 HistDigital APons MEiroa.pdf

17. Otzen T, Manterola C. Técnicas de muestreo sobre una población a estudio. Int. J. Morphol. [Internet]. 2017 [citado 20 de febrero de 2021];35(1):227-232. Disponible en: https://scielo.conicyt.cl/pdf/ijmorphol/v35n1/ar t37.pdf

18. Rodríguez Jiménez $A$, Pérez Jacinto $A O$. Métodos científicos de indagación y de construcción del conocimiento. Rev Esc Adm Neg [internet]. 2017 [citado 20 febrero 2021];(82):179-200. Disponible en: http://www.scielo.org.co/pdf/ean/n82/01208160-ean-82-00179.pdf

19. Ortiz Coss B. Partería en la Nueva España del siglo XVI al XVIII: influencias de la práctica europea. [Tesis de doctorado en internet]. Zacatecas: Universidad Autónoma de Zacatecas Francisco García Salinas;2018; [citado 13 abril 2021]. Disponible en: https://core.ac.uk/download/pdf/323141125.p df

20. Bazán Días I. El estupro: sexualidad delictiva en la Baja Edad Media y primera Edad Moderna. Mélanges de la Casa de Velázquez. [Internet]. 2003 [citado 13 abril 2021];33(1):13-46. Disponible en: https://addi.ehu.es/handle/10810/7988
21. Riva BC. El perito médico en los delitos sexuales, 1880-1890. En: Barrenche O, Bisso A, compiladores. Ayer, hoy y mañana son contemporáneo. Tradiciones, leyes y proyectos en América Latina. Argentina: EDULP; 2019. pp.229-258

22. Arias, J. Causa criminal contra Manuel Ximénez por el estupro violento intentado contra Severina Pineda, Goascorán. 1816. 20 p. Ubicado en: Archivo Nacional de Honduras, Fondo Colonial, Tegucigalpa, Honduras; documento 3863, caja 120.

23. Zelaya, F. Causa criminal contra Román Flores por estupro violento con Ignacia Romero, Tegucigalpa. 1819. 46 p. Ubicado en: Archivo Nacional de Honduras, Fondo de la Alcaldía Mayor de Tegucigalpa. Honduras; documento 5094, caja 141.

24. Muriel J. Hospitales de la Nueva España. México: Universidad Nacional Autónoma de México; 1990.

25. Ampudia J. Plano que demuestra la situación, y detalle que contiene el Hospital de San Juan de Dios de la Ciudad de Comayagua. 1783. 1 p. Ubicado en: Archivo General de Indias, Sevilla, España, signatura MP-Guatemala,250.

26. García E. Política y Estado en la sociedad hondureña del siglo XIX (1838-1872). Honduras: Instituto Hondureño de Antropología e Historia; 2008.

27. Fasano JP. Los delitos contra el honor en la 
justicia del crimen. Buenos Aires, 1840-1860. V Jornadas de Sociología de la UNLP. [Internet]. 2008 [citado 6 abril 2021]. Disponible en: http://www.memoria.fahce.unlp.edu.ar/trab e ventos/ev.6034/ev.6034.pdf

28. Alfaro Alfaro N, Villaseñor Farias M, Valadez Figueroa I, Guzmán Sánchez A, González Torres YS. Algunos aspectos históricos de la atención al embarazo. Investigación en Salud. [Internet]. 2006 [citado 20 febrero 2021];8(1):50-53. Disponible

en: http://repositorio.cualtos.udg.mx:8080/jspui/bi tstream/123456789/366/1/Algunos\%20aspecto s\%20históricos\%20de\%20la\%20atención\%20al\% 20embarazo.pdf

29. Dougnac Rodríguez A. Manual de historia del derecho indiano. 2a . ed. México: Universidad Nacional Autónoma de México; 1994.

30. Sierra Zelaya GM. Cámara de Gessell como herramienta investigativa en los abusos sexuales de niños y niñas. Caso de Honduras. Gac. int. cienc. forense. [Internet]. 2013 [citado en 13 de abril de 2021];(7):46-58. Disponible en: https://roderic.uv.es/bitstream/handle/10550/ 37830/4214052.pdf?sequence $=1$

31. Mantilla S. La revictimización como causal del silencio de la víctima. Rev. cienc. forenses Honduras. [Internet]. 2015 [citado 13 abril 2021];1(2):3-12. Disponible en: http://www.bvs.hn/RCFH/pdf/2015/pdf/RCFH12-2015-4.pdf

32. Centro de Derechos de Mujeres. Violencia sexual en Honduras: el impacto de las políticas de negación de los derechos de las mujeres. Observatorio de derechos humanos de las mujeres [Internet]. 2018 [citado 13 abril 2021];(11):1-16 Disponible en: http://derechosdelamujer.org/wpcontent/uploads/2018/11/Boletiěn-violenciasexual-WEB.pdf 